\title{
Commutativity Criterions Using Normal Subgroup Lattices
}

\author{
Simion BREAZ (*)
}

ABstRact - We prove that a group $G$ is Abelian whenever (1) it is nilpotent and the lattice of normal subgroups of $G$ is isomorphic to the subgroup lattice of an Abelian group or (2) there exists a non-torsion Abelian group $B$ such that the normal subgroup lattice of $B \times G$ is isomorphic to the subgroup lattice of an Abelian group. Using (2), it is proved that an Abelian group $A$ can be determined in the class of all groups by the lattice of all normal subgroups of some groups, e.g. if $A$ is an Abelian group and $G$ is a group such that $Z \times A$ and $Z \times G$ have isomorphic normal subgroup lattices then $A$ and $G$ are isomorphic groups.

\section{Introduction.}

If $G$ is a group, we will denote by $\mathcal{L}(G)$ the lattice of all subgroups of $G$ and by $\mathcal{N}(G)$ the lattice of all normal subgroups of $G$. It is well known that there are many important properties of a group $G$ which cannot be recovered from properties of $\mathcal{L}(G)$ or $\mathcal{N}(G)$. One of the simplest example is the following: $G$ is a simple group if and only if $\mathcal{N}(G)$ is a chain with 2 elements, so if we have a group $G$ with such a normal subgroup lattice we cannot say if $G$ is Abelian. However, there exist Abelian groups $A$ such that it is possible to deduce $G \cong A$ from the existence of an isomorphism $\mathcal{N}(G) \cong \mathcal{N}(A)$, by the works of Curzio, [4], and Brandl, [2]. Moreover, it is proved in [9] and [3] that subgroup lattices can be used to conclude that $G$ is Abelian: if $\mathcal{L}(G \times G)$ or $\mathcal{L}(Z \times G)$ are modular lattices then $G$ is Abelian. In particular, if $\mathcal{L}(G \times G) \cong \mathcal{L}(A)$ or $\mathcal{L}(Z \times G) \cong \mathcal{L}(A)$ for some Abelian group

(*) Indirizzo dell'A.: Babeş-Bolyai University, Faculty of Mathematics and Computer Science, Str. Mihail Kogălniceanu 1, 400084 Cluj-Napoca, Romania.

E-mail: bodo@math.ubbcluj.ro

1991 Mathematics Subject Classification. 20E15; 20K27; $20 \mathrm{~F} 18$.

The author is supported by the grant PN-II-CD ID-489 
$A$ then $G$ is an Abelian group. A natural question is if we can replace in these results the subgroup lattice with the normal subgroup lattice.

First we observe that this transfer cannot be done in all cases. For example, if $G$ is a non-abelian simple group, then it is superperfect (i.e. $[N, G]=N$ for all $N \unlhd G$ ). Then [10, Theorem 1] shows that $\mathcal{N}(G \times G) \cong\{1<G\} \times\{1<G\} \cong \mathcal{N}(Z / 2 Z \times Z / 3 Z)$ (direct products of two chains with two elements).

In this paper we will prove that some criterions for the commutativity of a group $G$ can be stated using normal subgroup lattices (Theorem 2 and Theorem 6): A group $G$ is Abelian if and only if one of the following conditions are satisfied:

(1) $\mathcal{N}(G) \cong \mathcal{N}(A)$ for an Abelian group $A$ and $G$ is nilpotent;

(2) $\mathcal{N}(B \times G) \cong \mathcal{N}(A)$ for some Abelian groups $B$ and $A$ such that $B$ is not a torsion group.

In the end of the paper we will discuss cancellation properties which can be deduced from (2).

\section{Direct products.}

If $G_{i}, i \in I$, is a family of groups, we will denote by $\prod_{i \in I}^{<\omega} G_{i}$ the restricted direct product of this family, i.e. the subgroup of all elements with finite support of the cartesian product $\prod_{i \in I} G_{i}$ (see [8, pp.118-119]). If all $G_{i}$ are Abelian then $\prod_{i \in I}^{<\omega} G_{i}$ is denoted by $\bigoplus_{i \in I} G_{i}$, and it is called the direct sum of the family $G_{i}, i \in I$. We note that, in order to simplify the exposition, we will identify every group $G_{i}$ with the corresponding subgroup of $\prod_{i \in I}^{<\omega} G_{i}$.

A well known theorem of Suzuki [12, Theorem 1.6.5] says us that the subgroup lattice $\mathcal{L}(G)$ of a group $G$ is a direct product of a family of lattices if and only if $G=\prod_{i \in I}^{<\infty} G_{i}$ such that the groups $G_{i}, i \in I$, are coprime, i.e. every $G_{i}$ is a torsion group and $\operatorname{gcd}\left(o\left(g_{i}\right), o\left(g_{j}\right)\right)=1$ for all $i \neq j, g_{i} \in G_{i}$ and $g_{j} \in G_{j}$ (here $o(g)$ denotes the order of the element $g$ ). There exists a version of this theorem for normal subgroup lattices. It was proved for the case $I$ is finite by Miller in [10, Theorem 2]. The hypothesis " $I$ is finite" is not essential in Millers's proof. However, for reader's convenience, we include a proof of this theorem. In order to enunciate it, let us recall that a group $G$ is superperfect if $[N, G]=N$ for every normal subgroup $N$ of $G$. 
THeOREm 1. Let $G$ be a group. The normal subgroup lattice of $G$ has a direct decomposition $\mathcal{N}(G)=\prod_{i \in I} L_{i}$ if and only if $G=\prod_{i \in I}^{<\omega} G_{i}$ such that

(a) at most one of the groups $G_{i}$ is not superperfect or

(b) for every family of normal subgroups $H_{i} \unlhd G_{i}$, the groups $H_{i} /\left[H_{i}, G_{i}\right], i \in I$, are coprime.

In this case $\mathcal{N}\left(G_{i}\right)=L_{i}$ for all $i \in I$.

Proof. Suppose that $\mathcal{N}(G)=\prod_{i \in I} L_{i}$. For every $j \in I$ we denote by $0_{j}$ and $1_{j}$ the least, respectively the greatest, element of $L_{i}$. We denote by $\overline{1}_{j}=\left(\ell_{i}\right)_{i} \in \mathcal{N}(G)$ the element defined by conditions $\ell_{i}=0_{i}$ for all $i \neq j$ and $\ell_{j}=1_{j}$. For every $j \in I$ we consider the normal subgroups $G_{j}$ of $G$ which correspond to $\overline{1}_{j}$. Then for every $j \neq k \in I$ the normal subgroups $G_{j}$ and $G_{k}$ centralize each other since $G_{j} \cap G_{k}=\{1\}$. Moreover, for every $j \in I$ we have $G=G_{j}\left\langle\bigcup_{i \neq j} G_{i}\right\rangle$ and $G_{j} \cap\left\langle\bigcup_{i \neq j} G_{i}\right\rangle=\{1\}$, hence $G=\prod_{i \in I}^{<\omega} G_{i}$. Moreover, since $\mathcal{N}(G)=\prod_{i \in I} \mathcal{N}\left(G_{i}\right)$, it follows that every normal subgroup $N$ of $G$ is a direct product of normal subgroups $N_{i}$ of the groups $G_{i}$.

Suppose that there exists $j \neq k$ in $I$ such that $G_{j}$ and $G_{k}$ are not superperfect groups. Then there are normal subgroups $H_{j} \unlhd G_{j}$ and $H_{k} \unlhd G_{k}$ such that $H_{j} \neq\left[H_{j}, G_{j}\right]$ and $H_{k} \neq\left[H_{k}, G_{k}\right]$. If the Abelian groups $H_{j} /\left[H_{j}, G_{j}\right]$ and $H_{k} /\left[H_{k}, G_{k}\right]$ are not coprime then, by Suzuki's Theorem, there exists a subgroup

$$
\bar{N} \leq H_{j} /\left[H_{j}, G_{j}\right] \times H_{k} /\left[H_{k}, G_{k}\right]
$$

which is not a direct product of type $\bar{K}_{j} \times \bar{K}_{k}$ with $\bar{K}_{j} \leq H_{j} /\left[H_{j}, G_{j}\right]$ and $\bar{K}_{k} \leq H_{k} /\left[H_{k}, G_{k}\right]$. Then the subgroup $N \leq H_{j} \times H_{k}$, which is determined by the properties $\left[H_{j}, G_{j}\right] \times\left[H_{k}, G_{k}\right] \leq N$ and $\bar{N}=N /\left(\left[H_{j}, G_{j}\right] \times\left[H_{k}, G_{k}\right]\right)$, is not a direct product of type $K_{j} \times K_{k}$ with $K_{j} \leq H_{j}$ and $K_{k} \leq H_{k}$, a contradiction.

Conversely, let $N$ be a normal subgroup of $G$, and $N_{i}=\pi_{i}(N) \unlhd G_{i}$, where $\pi_{i}: G \rightarrow G_{i}$ are the canonical projections, for all $i \in I$. Then

$$
\prod_{i \in I}^{<\omega}\left[N_{i}, G_{i}\right] \leq N \leq \prod_{i \in I}^{<\omega} N_{i}
$$

If we are under the hypothesis (a), so there exists $j \in I$ such that all groups 
$G_{i}(i \neq j)$ are superperfect, then $N=N_{j} \times \prod_{i \neq j}^{<\omega} N_{i}$. If the hypothesis (b) is satisfied, then we can apply again Suzuki's Theorem to observe that $N / \prod_{i \in I}^{<\omega}\left[N_{i}, G_{i}\right]=\prod_{i \in I}^{<\omega} K_{i} /\left[N_{i}, G_{i}\right]$ for some normal subgroups $K_{i} \unlhd N_{i}$, hence $N=\prod_{i \in I}^{<\omega} K_{i}$, and the proof is complete.

\section{Commutativity criterions.}

Using Theorem 1, we can prove a criterion for the commutativity of a nilpotent group.

THEOREM 2. A nilpotent group $G$ is Abelian if and only if there exists an abelian group $A$ such that $\mathcal{N}(G) \cong \mathcal{N}(A)$.

Proof. Let $\varphi: \mathcal{N}(G) \rightarrow \mathcal{N}(A)$ be an isomorphism.

Suppose that $G$ is not abelian. Then, by [12, Theorems 9.1.12, 9.1.14], $A$ is a torsion group. We decompose $A$ as a direct sum of its $p$-components: $A=\bigoplus_{p} A_{p}$. Then $G=\prod_{p}^{<\omega} \varphi^{-1}\left(A_{p}\right)$, as a consequence of Theorem 1. It follows that there exists a prime $p$ such that $G_{p}=\varphi^{-1}\left(A_{p}\right)$ is not Abelian.

Therefore we can suppose that $A$ is a $p$-group. Using [12, Theorem 9.1.11], we observe that $A$ is locally cyclic, hence its subgroup lattice is a chain by [5, Theorem 3.1]. Therefore $\mathcal{N}(G)=\left\{1<N_{1}<\cdots<N_{k}<\cdots\right\}$. Let $c \in \mathbb{N}$ be the nilpotency class of $G$. Since $G$ is not Abelian, $c \geq 2$. If $Z_{i}, i \in\{0, \ldots c\}$, denotes the terms of the upper central series of $G$, then there exists $k$ such that $Z_{c-1}=N_{k}$. Hence in the group $H=G / Z_{c-2}$, we have the properties: $Z(H)=Z_{c-1} / Z_{c-2}$, and $H / Z(H)$ is abelian. Moreover, every subgroup of $H / Z(H)$ is of the form $\left(N / Z_{c-2}\right) / Z(H)$, with $N$ normal in $G$. Hence the subgroup lattice of the Abelian group $H / Z(H)$ is a chain, hence $H / Z(H)$ is a union of an ascending chain of cyclic subgroups (see [5, Section 3]).

If $x, y \in H$ then there exists $u \in H$ such that $x Z(H), y Z(H) \in\langle u Z(H)\rangle$. Therefore $x=u^{k} v$ and $y=u^{\ell} z$ for some integers $k$ and $\ell$ and some elements $v, z \in Z(H)$. Then $x y=u^{k+\ell} v z=y x$, hence $H$ is abelian. It follows that $Z_{c-1}=G$, a contradiction. Therefore $G$ is abelian.

EXAMPLE 3. The previous result is not valid for solvable groups, since the lattices $\mathcal{N}\left(S_{3}\right)$ and $\mathcal{N}\left(Z / 2^{2} Z\right)$ are chains with 3 elements, hence they are isomorphic. 
COROLLARY 4. If $G$ is a finite p-group such that $\mathcal{N}(G) \cong \mathcal{N}(A)$ for some Abelian group A, then $G$ is Abelian.

REMARK 5. a) Corollary 4 does not work for subgroup lattices of finite $p$-groups, as a consequence of [12, Theorem 2.5.9].

b) Since there exists infinite simple $p$-groups, Corollary 4 is not valid for general $p$-groups.

However, we can enunciate a result which is valid for all groups.

Theorem 6. Let B be an Abelian group which is not a torsion group. A group $G$ is Abelian if and only if there exists an abelian group $A$ such that $\mathcal{N}(B \times G) \cong \mathcal{N}(A)$.

Proof. Let $\varphi: \mathcal{N}(B \times G) \cong \mathcal{N}(A)$ be an isomorphism. Since every subgroup of $B$ is normal in $B \times G$, the restriction of $\varphi$ to $B$ induces a projectivity from $B$ onto $\varphi(B)$ (i.e. $\varphi_{\mid \mathcal{L}(B)}: \mathcal{L}(B) \rightarrow \mathcal{L}(\varphi(B))$ is an isomorphism). Then $\mathcal{L}(\varphi(B))$ is not an atomic lattice (i.e. there exists an element which is not minimized by an atom), hence $\varphi(B)$ is not a torsion group. Therefore, $A$ is not a torsion group.

If the torsion-free rank of $A$ is $>1$ then $A \cong B \times G$ as a consequence of [12, Theorem 9.1.12].

If $A$ is of torsion-free rank 1 , using the direct decomposition $A=\varphi(B) \times \varphi(G)$, we observe that $\varphi(G)$ is a torsion group. For every element $x \in A$ of infinite order we know that $\langle x\rangle$ and the torsion part $T(A)$ of $A$ are disjoint (normal) subgroups of $A$. Hence $\varphi^{-1}(\langle x\rangle)$ and $G$ are disjoint normal subgroups of $B \times G$, and it follows that $G$ is a subgroup of the centralizer of $\varphi^{-1}(\langle x\rangle)$ for all $x \in A \backslash T(A)$. Therefore, $G$ is contained in the center of $B \times G$, since

$$
B \times G=\varphi^{-1}(A)=\varphi^{-1}\left(\vee_{x \in A \backslash T(A)}\langle x\rangle\right)=\vee_{x \in A \backslash T(A)} \varphi^{-1}(\langle x\rangle),
$$

hence $G$ is Abelian.

EXAMPLE 7. The hypothesis " $B$ is not a torsion group" is essential in the previous theorem.

Proof. Let $B=Z / 5 Z$ and $G=S_{3}$. Using Theorem 1 we deduce that

$$
\mathcal{N}(B \times G) \cong \mathcal{N}(B) \times \mathcal{N}(G)=\{0<B\} \times\left\{1<A_{3}<S_{3}\right\} .
$$

But this lattice is isomorphic to the subgroup lattice of $Z / 5 Z \times Z / 2^{2} Z$. 
REMARK 8. As a consequence of [12, Theorem 9.1.11], the property stated in the previous theorem is valid if we assume that all involved groups are $p$-groups.

However, if only $B \neq 1$ and $G$ are $p$-groups the property is not valid. To see this, it is enough to consider $B=Z / p Z, G$ an infinite simple $p$-group and $A=Z / p Z \times Z / q Z$ (where $q \neq p$ are prime integers), and to apply Theorem 1 .

It is proved in [3] that every abelian group is determined in the class of all groups by some subgroup lattices. As a consequence of Theorem 6 , we obtain similar results for normal subgroup lattices.

CoRollary 9. Let $G$ be a group.

a) If $B \neq 1$ is a torsion-free Abelian group, and $T$ is a torsion Abelian group such that $\mathcal{N}(B \times T) \cong \mathcal{N}(B \times G)$, then $T \cong G$.

b) If $A$ is an Abelian group and $B \neq 1$ is a finite rank torsion-free Abelian group such that $\mathcal{N}(B \times A) \cong \mathcal{N}(B \times G)$, then there exists a positive integer $n$ such that $A^{n} \cong G^{n}$.

c) If $A$ is an Abelian group and $\mathcal{N}(Z \times A) \cong \mathcal{N}(Z \times G)$ then $A \cong G$.

d) If $A$ is an Abelian group and $\mathcal{N}(\mathrm{Q} \times A) \cong \mathcal{N}(\mathrm{Q} \times G)$ then $A \cong G$.

Proof. We know that $G$ is Abelian by Theorem 6 .

For a) we apply [12, Theorem 2.6.10 and Theorem 2.6.15].

For b) we use a) and [12, Theorem 2.6.10] to deduce $B \times A \cong B \times G$. Then we apply the "power cancellation property" proved by Goodearl in $[6$, Theorem 5.1].

The proofs for c) and d) are similar, using this time the fact that Z and $\mathrm{Q}$ are cancellable from direct products of Abelian groups (see [1, Corollary 8.8]).

REMARK 10. As a consequence of [13, Theorem 13], there exist nonisomorphic groups $G$ and $H$ such that $Z \times G \cong Z \times H$. Then Corollary $9 \mathrm{c}$ ) cannot be extended to general groups. However, if $Z \times G \cong Z \times H$ for some non-isomorphic groups $G$ and $H$, these groups must be infinite, as a consequence of [13, Theorem 11], so a natural question is if we can extend Corollary 9 to non-Abelian finite groups.

The answer for this question is negative, and it is based on a (negative) solution which was found by Kearnes and Szendrei [7] for the problem formulated in [11]: "can we deduce $G \cong H$ from $\mathcal{L}\left(G^{n}\right) \cong \mathcal{L}\left(H^{n}\right)$ for all $n \geq 1$ ?". 
To present this answer, let us recall that two groups $(G, \cdot)$ and $(G, \circ)$, defined on the same set $G$, are term equivalent if they have the same term functions. Consequently, if $(G, \cdot)$ and $(G, \circ)$ are term equivalent groups then

(T1) they have the same identity, and

(T2) the operations - and $\circ$ are term operations with respect to $\circ$ and ·, respectively.

We recall that a term operation with respect to $\cdot$ is a function

$$
G \times G \rightarrow G,(x, y) \mapsto T(x, y)=x^{m_{1}} \cdot y^{n_{1}} \cdots x^{m_{\ell}} \cdot y^{n_{\ell}} \text { for all } x, y \in G,
$$

where $T=X^{m_{1}} Y^{n_{1}} \cdots X^{m_{\ell}} Y^{n_{\ell}}$ is a binary term $\left(m_{i}, n_{i} \in Z\right.$ for all $\left.i\right)$.

The proof of the following lemma is included in the proof of $[7$, Lemma 2.4].

LEMma 11. Let $(G, \cdot)$ be a group and $T$ a binary term. If the pair $(G, \circ)$, where $x \circ y=T(x, y)$, is a group with the same identity as $(G, \cdot)$ then there exist $r_{1}, \ldots, r_{k} \in Z, s_{1}, \ldots, s_{k} \in Z$ and $\varepsilon_{1}, \ldots, \varepsilon_{k} \in\{ \pm 1\}$ such that

$$
\text { (甘) } T(x, y)=x y\left[x^{r_{1}}, y^{s_{1}}\right]^{\varepsilon_{1}} \ldots\left[x^{r_{k}}, y^{s_{k}}\right]^{\varepsilon_{k}}
$$

for all $x, y \in G$ (here $\left.[a, b]=a b a^{-1} b^{-1}\right)$.

Proof. There exist integers $m_{1}, \ldots, m_{k}, n_{1}, \ldots, n_{k}$ such that we can write $T(x, y)=x y x^{m_{1}} y^{n_{1}} \cdots x^{m_{k}} y^{n_{k}}$ for all $x, y \in G$. From $T(x, 1)=x$, respectively $T(1, y)=y$, for all $x, y \in G$, we deduce

$$
x^{m_{1}+\ldots+m_{k}}=y^{n_{1}+\ldots+n_{k}}=1 .
$$

Moreover, for all $x, y \in G$ we have

$$
\begin{aligned}
T(x, y) & =x y x^{m_{1}} y^{n_{1}} \cdots x^{m_{k}} y^{n_{k}} \\
& =x y\left[x^{m_{1}}, y^{n_{1}}\right] y^{n_{1}} x^{m_{1}+m_{2}} y^{n_{2}} \cdots x^{m_{k}} y^{n_{k}}=\cdots \\
& =x y\left[x^{m_{1}}, y^{n_{1}}\right]\left[x^{m_{1}+m_{2}}, y^{n_{1}}\right]^{-1} x^{m_{1}+m_{2}} y^{n_{1}+n_{2}} \cdots,
\end{aligned}
$$

and so on, hence the function $T(x, y)$ has the form ( $\sharp)$.

REMARK 12. If $G=(G, \cdot)$ and $H=(G, \circ)$ are groups as in Lemma 11, then for every $x \in G$, its inverse $x^{-1}$ in $G$ is the inverse of $x$ in $H$. Therefore, to prove that two groups $G=(G, \cdot)$ and $H=(G, \circ)$ are term equivalent, it is enough to prove that they satisfy the conditions (T1) and (T2). 
CoRollary 13. If $G=(G, \cdot)$ and $H=(G, \circ)$ are term equivalent groups, then $Z \times G$ and $Z \times H$ are term equivalent groups.

Proof. By Lemma 11, there is a binary term

$$
T=X Y\left[X^{r_{1}}, Y^{s_{1}}\right]^{\varepsilon_{1}} \cdots\left[X^{r_{k}}, Y^{s_{k}}\right]^{\varepsilon_{k}}
$$

with $r_{1}, \ldots, r_{k} \in Z, s_{1}, \ldots, s_{k} \in Z$ and $\varepsilon_{1}, \ldots, \varepsilon_{k} \in\{ \pm 1\}$ such that the operation $\circ$ is the term function induced by $T$.

If we consider the term function induced by $T$ on $Z \times G$ we observe that $T((m, x),(n, y))=(m+n, x \circ y)$ for all $m, n \in Z$ and $x, y \in G$. Then the operation on $Z \times H$ is a term operation on the set $Z \times G$ with respect to the operation of the group $Z \times G$. By symmetry, the operation on $Z \times G$ is also a term operation with respect to the operation of $Z \times H$. It is not hard to see that the identities of the groups $Z \times G$ and $Z \times H$ are the same. Therefore, the groups $Z \times G$ and $Z \times H$ are term equivalent.

COROLLARY 14. There exist non-isomorphic finite groups $G$ and $H$ such that the lattices $\mathcal{N}(Z \times G)$ and $\mathcal{N}(Z \times H)$ are isomorphic.

Proof. By [7, Example 2.14], there exist non-isomorphic term equivalent finite groups $G$ and $H$. Then $Z \times G$ and $Z \times H$ are non-isomorphic term equivalent groups. Applying [7, Lemma 2.6], we obtain $\mathcal{N}(Z \times G)=\mathcal{N}(Z \times H)$.

Acknowledgments. I would like to thank the referee for his/her careful reading of the paper and helpful critical remarks and suggestions.

\section{REFERENCES}

[1] D. ARnold, Finite Rank Torsion-Free Abelian Groups and Rings, Lecture Notes in Math., 931 (Springer - Verlag, New-York, 1982).

[2] R. BRandL, On groups with certain lattices of normal subgroups, Arch. Math. (Basel), 47 (1986), pp. 6-11.

[3] S. Breaz - Gr. Călugăreanu, Every Abelian group is determined by a subgroup lattice, Stud. Sci. Math. Hung., 45 (2008), pp. 135-137.

[4] M. CuRzIo, Una caratterizzazione reticolare dei gruppi abeliani, Rend. Math. e. Appl., 24 (1965), pp. 1-10.

[5] L. Fuchs, Infinite Abelian Groups, vol. I, Academic Press, New-York and London, 1970.

[6] K. GOODEARL, Power cancellation of groups and modules, Pacific J. Math., 64 (1976), pp. 387-411. 
[7] K. A. KeARnEs - Á. SzEndREI, Groups with identical subgroup lattices in all powers, J. Group Theory, 7 (2004), pp. 385-402.

[8] A. G. Kurosh, The Theory of Groups, Chelsea Publishing Company (NewYork, 1960).

[9] E. LuKÁCS - P. P. PÁLFY, Modularity of the subgroup lattice of a direct square, Arch. Math. (Basel), 46 (1986), pp. 18-19.

[10] M. D. Miller, On the lattice of normal subgroups of a direct product, Pacific J. Math., 60 (1975), pp. 153-158.

[11] P. P. PÁlfy, Groups and Lattices, Groups St. Andrews 2001 in Oxford. Vol. II, London Math. Soc. Lecture Note Ser., 305, Cambridge Univ. Press (Cambridge, 2003), pp. 428-454.

[12] R. SchmidT, Subgroup Lattices of Groups, de Gruyter Expositions in Mathematics 14, de Gruyter (Berlin, 1994).

[13] E. A. Walker, Cancellation in direct sums of groups, Proc. A.M.S., 7 (1956), pp. 898-902.

Manoscritto pervenuto in redazione il 28 novembre 2008. 
